\title{
Generalized Growth of Special Monogenic Functions
}

\author{
Susheel Kumar \\ Department of Mathematics, Jaypee University of Information Technology, Samirpur 177601 (H.P.), India
}

Correspondence should be addressed to Susheel Kumar; sus83dma@gmail.com

Received 6 January 2014; Accepted 2 March 2014; Published 1 April 2014

Academic Editor: Yan Xu

Copyright (C) 2014 Susheel Kumar. This is an open access article distributed under the Creative Commons Attribution License, which permits unrestricted use, distribution, and reproduction in any medium, provided the original work is properly cited.

We study the generalized growth of special monogenic functions. The characterizations of generalized order, generalized lower order, generalized type, and generalized lower type of special monogenic functions have been obtained in terms of their Taylor's series coefficients.

\section{Introduction}

Clifford analysis offers the possibility of generalizing complex function theory to higher dimensions. It considers Clifford algebra valued functions that are defined in open subsets of $\mathbb{R}^{n}$ for arbitrary finite $n \in \mathbb{N}$ and that are solutions of higherdimensional Cauchy-Riemann systems. These are often called Clifford holomorphic or monogenic functions.

In order to make calculations more concise, we use the following notations, where $\mathbf{m}=\left(m_{1}, \ldots, m_{n}\right) \in \mathbb{N}_{0}^{n}$ is $n$ dimensional multi-index and $\mathbf{x} \in \mathbb{R}^{n}$ :

$$
\begin{aligned}
\mathbf{x}^{\mathbf{m}} & =x_{1}^{m_{1}}, \ldots, x_{n}^{m_{n}}, \\
\mathbf{m} ! & =m_{1} !, \ldots, m_{n} !, \\
|\mathbf{m}| & =m_{1}+\cdots+m_{n} .
\end{aligned}
$$

Following Almeida and Kraußhar [1] and Constales et al. [2, $3]$, we give some definitions and associated properties.

By $\left\{e_{1}, e_{2}, \ldots, e_{n}\right\}$ we denote the canonical basis of the Euclidean vector space $\mathbb{R}^{n}$. The associated real Clifford algebra $\mathrm{Cl}_{0 n}$ is the free algebra generated by $\mathbb{R}^{n}$ modulo $\mathbf{x}^{2}=-\|\mathbf{x}\|^{2} e_{0}$, where $e_{0}$ is the neutral element with respect to multiplication of the Clifford algebra $\mathrm{Cl}_{0 n}$. In the Clifford algebra $\mathrm{Cl}_{0 n}$, the following multiplication rule holds:

$$
e_{i} e_{j}+e_{j} e_{i}=-2 \delta_{i j} e_{0}, \quad i, j=1,2, \ldots, n,
$$

where $\delta_{i j}$ is Kronecker symbol. A basis for Clifford algebra $\mathrm{Cl}_{0 n}$ is given by the set $\left\{e_{A}: A \subseteq\{1,2, \ldots, n\}\right\}$ with $e_{A}=$ $e_{l_{1}} e_{l_{2}} \ldots e_{l_{r}}$, where $1 \leq l_{1}<l_{2}<\cdots<l_{r} \leq n, e_{\phi}=e_{0}=1$.
Each $a \in \mathrm{Cl}_{0 n}$ can be written in the form $a=\sum_{A} a_{A} e_{A}$ with $a_{A} \in \mathbb{R}$. The conjugation in Clifford algebra $\mathrm{Cl}_{0 n}$ is defined by $\bar{a}=\sum_{A} a_{A} \bar{e}_{A}$, where $\bar{e}_{A}=\bar{e}_{l_{r}} \bar{e}_{l_{r-1}}, \ldots, \bar{e}_{l_{1}}$ and $\bar{e}_{j}=-e_{j}$ for $j=1,2, \ldots n, \bar{e}_{0}=e_{0}=1$. The linear subspace $\operatorname{span}_{R}\left\{1, e_{1}, \ldots, e_{n}\right\}=\mathbb{R} \oplus \mathbb{R}^{n} \subset \mathrm{Cl}_{0 n}$ is the so-called space of paravectors $z=x_{0}+x_{1} e_{1}+x_{2} e_{2}+\cdots+x_{n} e_{n}$ which we simply identify with $\mathbb{R}^{n+1}$. Here, $x_{0}=\mathrm{Sc}(z)$ is scalar part and $\mathbf{x}=x_{1} e_{1}+x_{2} e_{2}+\cdots+x_{n} e_{n}=\operatorname{Vec}(z)$ is vector part of paravector $z$. The Clifford norm of an arbitrary $a=\sum_{A} a_{A} e_{A}$ is given by

$$
\|a\|=\left(\sum_{A}\left|a_{A}\right|^{2}\right)^{1 / 2} .
$$

Also, for $b \in \mathrm{Cl}_{0 n}$, we have $\|a b\| \leq 2^{n / 2}\|a\|\|b\|$. Each paravector $z \in \mathbb{R}^{n+1} \backslash\{0\}$ has an inverse element in $\mathbb{R}^{n+1}$ which can be represented in the form $z^{-1}=\bar{z} /\|z\|^{2}$. In order to make calculations more concise, we use the following notation:

$$
\begin{gathered}
k_{\mathbf{m}}=\left(\begin{array}{c}
n+|\mathbf{m}|-1 \\
|\mathbf{m}|
\end{array}\right)=\frac{(n)_{\mathbf{m}}}{\mathbf{m} !}, \\
(n)_{\mathbf{m}}=n(n+1), \ldots,(n+|\mathbf{m}|-1) .
\end{gathered}
$$

The generalized Cauchy-Riemann operator in $\mathbb{R}^{n+1}$ is given by

$$
D \equiv \frac{\partial}{\partial x_{0}}+\sum_{i=1}^{n} e_{i} \frac{\partial}{\partial x_{i}}
$$


If $U \subseteq \mathbb{R}^{n+1}$ is an open set, then a function $g: U \rightarrow \mathrm{Cl}_{0 n}$ is called left (right) monogenic at a point $z \in U$ if $D g(z)=0$ $(g D(z)=0)$. The functions which are left (right) monogenic in the whole space are called left (right) entire monogenic functions.

Following Abul-Ez and Constales [4], we consider the class of monogenic polynomials $p_{\mathbf{m}}$ of degree $|\mathbf{m}|$, defined as

$$
p_{\mathbf{m}}(z)=\sum_{i+j=|\mathbf{m}|}^{\infty} \frac{((n-1) / 2)_{i}}{i !} \frac{((n+1) / 2)_{j}}{j !}(\bar{z})^{i} z^{j} .
$$

Let $w_{n}$ be $n$-dimensional surface area of $n+1$-dimensional unit ball and let $S^{n}$ be $n$-dimensional sphere. Then, the class of monogenic polynomials described in (6) satisfies (see [5], pp. 1259)

$$
\frac{1}{w_{\mathbf{m}}} \int_{S^{n}} \overline{p_{\mathbf{m}}(z)} p_{1}(z) d S_{z}=k_{\mathbf{m}} \delta_{|\mathbf{m}||\mathbf{1}|}
$$

Also following Abul-Ez and De Almeida [5], we have

$$
\max _{\|z\|=r}\left\|p_{\mathbf{m}}(z)\right\|=k_{\mathbf{m}} r^{\mathbf{m}}
$$

\section{Preliminaries}

Now following Abul-Ez and De Almeida [5], we give some definitions which will be used in the next section.

Definition 1. Let $\Omega$ be a connected open subset of $\mathbb{R}^{n+1}$ containing the origin and let $g(z)$ be monogenic in $\Omega$. Then, $g(z)$ is called special monogenic in $\Omega$, if and only if its Taylor's series near zero has the form (see [5], pp. 1259)

$$
g(z)=\sum_{|\mathbf{m}|=0}^{\infty} p_{\mathbf{m}}(z) c_{\mathbf{m}}, \quad c_{\mathbf{m}} \in \mathrm{Cl}_{0 n} \text {. }
$$

Definition 2. Let $g(z)=\sum_{|\mathbf{m}|=0}^{\infty} p_{\mathbf{m}}(z) c_{\mathbf{m}}$ be a special monogenic function defined on a neighborhood of the closed ball $B(0, r)$. Then,

$$
\left\|c_{\mathbf{m}}\right\| \leq \frac{1}{\sqrt{k_{\mathrm{m}}}} M(r, g) r^{-\mathbf{m}}
$$

where $M(r, g)=\max _{\|z\|=r}\|g(z)\|$ is the maximum modulus of $g(z)$ (see [5], pp. 1260).

Definition 3. Let $g: \mathbb{R}^{n+1} \rightarrow \mathrm{Cl}_{0 n}$ be a special monogenic function whose Taylor's series representation is given by (9). Then, for $r>0$ the maximum term of this special monogenic function is given by (see [5], pp. 1260)

$$
\mu(r)=\mu(r, g)=\max _{|\mathbf{m}| \geq 0}\left\{\left\|a_{\mathbf{m}}\right\| k_{\mathbf{m}} r^{\mathbf{m}}\right\} .
$$

Also the index m with maximal length $|\mathbf{m}|$ for which maximum term is achieved is called the central index and is denoted by (see [5], pp. 1260)

$$
\nu(r)=\nu(r, f)=\mathbf{m} .
$$

Definition 4. Let $g: \mathbb{R}^{n+1} \rightarrow \mathrm{Cl}_{0 n}$ be a special monogenic function whose Taylor's series representation is given by (9). Then, the order $\rho$ and lower order $\lambda$ of $g(z)$ are defined as (see [5], pp. 1263)

$$
\frac{\rho}{\lambda}=\lim _{r \rightarrow \infty} \sup _{\inf } \frac{\log \log M(r, g)}{\log r} .
$$

Definition 5. Let $g: \mathbb{R}^{n+1} \rightarrow \mathrm{Cl}_{0 n}$ be a special monogenic function whose Taylor's series representation is given by (9). Then, the type $\sigma$ and lower type $\omega$ of special monogenic function $g(z)$ having nonzero finite generalized order are defined as (see [5], pp. 1270)

$$
{ }_{\omega}^{\sigma}=\lim _{r \rightarrow \infty} \sup _{\inf } \frac{\log M(r, g)}{r^{\rho}} .
$$

For generalization of the classical characterizations of growth of entire functions, Seremeta [6] introduced the concept of the generalized order and generalized type with the help of general growth functions as follows.

Let $L^{0}$ denote the class of functions $h(x)$ satisfying the following conditions:

(i) $h(x)$ is defined on $[a, \infty)$ and is positive, strictly increasing, and differentiable, and it tends to $\infty$ as $x \rightarrow \infty$,

(ii) $\lim _{x \rightarrow \infty}(h[\{1+1 / \psi(x)\} x] / h(x))=1$, for every function $\psi(x)$ such that $\psi(x) \rightarrow \infty$ as $x \rightarrow$ $\infty$.

Let $\Lambda$ denote the class of functions $h(x)$ satisfying conditions (i) and

(i) $\lim _{x \rightarrow \infty}(h(c x) / h(x))=1$,

(ii) for every $c>0$; that is, $h(x)$ is slowly increasing.

Following Srivastava and Kumar [7] and Kumar and Bala ([8-10]), here we give definitions of generalized order, generalized lower order, generalized type, and generalized lower type of special monogenic functions. For special monogenic function $g(z)$ and functions $\alpha(x) \in \Lambda, \beta(x) \in L^{0}$, we define the generalized order $\rho(\alpha, \beta, g)$ and generalized lower order $\lambda(\alpha, \beta, g)$ of $g(z)$ as

$$
\begin{aligned}
& \rho(\alpha, \beta, g) \\
& \lambda(\alpha, \beta, g)
\end{aligned}=\lim _{r \rightarrow \infty} \sup \frac{\alpha[\log M(r, g)]}{\beta(\log r)} .
$$

If in above equation we put $\alpha(r)=\log r$ and $\beta(r)=r$, then we get definitions of order and lower order as defined by Abul-Ez and De Almeida (see [5], pp. 1263). Hence, their definitions of order and lower order are special cases of our definitions.

Further, for $\alpha(x), \beta^{-1}(x), \gamma(x) \in L^{0}$, we define the generalized type $\sigma(\alpha, \beta, \rho, g)$ and generalized lower type of special monogenic function $g(z)$ having nonzero finite generalized order as

$$
\begin{aligned}
& \sigma(\alpha, \beta, \rho, g) \\
& \omega(\alpha, \beta, \rho, g)
\end{aligned}=\lim _{r \rightarrow \infty} \sup _{\inf } \frac{\alpha[\log M(r, g)]}{\beta\left[\{\gamma(r)\}^{\rho}\right]} .
$$


If in above equation we put $\alpha(r)=r, \beta(r)=r$, and $\gamma(r)=r$, then we get definitions of type and lower type as defined by Abul-Ez and De Almeida (see [5], pp. 1270). Hence, their definitions of type and lower type are special cases of our definitions.

Abul-Ez and De Almeida [5] have obtained the characterizations of order, lower order, type, and lower type of special monogenic functions in terms of their Taylor's series coefficients. In the present paper we have obtained the characterizations of generalized order, generalized lower order, generalized type and generalized lower type of special monogenic functions in terms of their Taylor's series coefficients. The results obtained by Abul-Ez and De Almeida [5] are special cases of our results.

\section{Main Results}

We now prove the following.

Theorem 6. Let $g: \mathbb{R}^{n+1} \rightarrow \mathrm{Cl}_{0 n}$ be a special monogenic function whose Taylor's series representation is given by (9). If $\alpha(x) \in \Lambda$ and $\beta(x) \in L^{0}$, then the generalized order $\rho$ of $g(z)$ is given as

$$
\rho=\rho(\alpha, \beta, g)=\lim _{|\mathbf{m}| \rightarrow \infty} \sup \frac{\alpha(|\mathbf{m}|)}{\beta\left\{\log \left\|c_{\mathbf{m}}\right\|^{-1 /|\mathbf{m}|}\right\}} .
$$

Proof. Write

$$
\theta=\lim _{|\mathbf{m}| \rightarrow \infty} \sup \frac{\alpha(|\mathbf{m}|)}{\beta\left\{\log \left\|c_{\mathbf{m}}\right\|^{-1 /|\mathbf{m}|}\right\}} .
$$

Now, first we prove that $\rho \geq \theta$. The coefficients of a monogenic Taylor's series satisfy Cauchy's inequality; that is,

$$
\left\|c_{\mathbf{m}}\right\| \leq \frac{1}{\sqrt{k_{\mathbf{m}}}} M(r, g) r^{-|\mathbf{m}|} .
$$

Also from (15), for arbitrary $\varepsilon>0$ and all $r>r_{0}(\varepsilon)$, we have

$$
M(r, g) \leq \exp \left[\alpha^{-1}\{\bar{\rho} \beta(\log r)\}\right], \quad \bar{\rho}=\rho+\varepsilon .
$$

Now, from inequality (19), we get

$$
\left\|c_{\mathbf{m}}\right\| \leq \frac{1}{\sqrt{k_{\mathbf{m}}}} r^{-|\mathbf{m}|} \exp \left[\alpha^{-1}\{\bar{\rho} \beta(\log r)\}\right] .
$$

Since $\left(1 / \sqrt{k_{\mathrm{m}}}\right) \leq 1$, (see [11], pp. 148) so the above inequality reduces to

$$
\left\|c_{\mathbf{m}}\right\| \leq r^{-|\mathbf{m}|} \exp \left[\alpha^{-1}\{\bar{\rho} \beta(\log r)\}\right]
$$

Putting $r=\exp \left[\beta^{-1}\{\alpha(|\mathbf{m}|) / \bar{\rho}\}\right]$ in the above inequality, we get, for all large values of $|\mathbf{m}|$,

$$
\left\|c_{\mathbf{m}}\right\| \leq \exp \left[|\mathbf{m}|-|\mathbf{m}| \beta^{-1}\left\{\frac{\alpha(|\mathbf{m}|)}{\bar{\rho}}\right\}\right]
$$

or

$$
\beta^{-1}\{\alpha(|\mathbf{m}|) / \bar{\rho}\} \leq 1-\frac{1}{|\mathbf{m}|}\left\{\log \left\|c_{\mathbf{m}}\right\|\right\}
$$

or

$$
\frac{\alpha(|\mathbf{m}|)}{\beta\left\{1+\log \left\|c_{\mathbf{m}}\right\|^{-1 /|\mathbf{m}|}\right\}} \leq \bar{\rho}
$$

or

$$
\frac{\alpha(|\mathbf{m}|)}{\beta\left\{\log \left\|c_{\mathbf{m}}\right\|^{-1 /|\mathbf{m}|}\right\}} \leq \bar{\rho} \frac{\beta\left\{1+\log \left\|c_{\mathbf{m}}\right\|^{-1 /|\mathbf{m}|}\right\}}{\beta\left\{\log \left\|c_{\mathbf{m}}\right\|^{-1 /|\mathbf{m}|}\right\}} .
$$

Since $\beta(x) \in L^{0}, \beta(1+x) \simeq \beta(x)$. Hence, proceeding to limits as $|\mathbf{m}| \rightarrow \infty$, we get

$$
\theta=\lim _{|\mathbf{m}| \rightarrow \infty} \sup \frac{\alpha(|\mathbf{m}|)}{\beta\left\{\log \left\|c_{\mathbf{m}}\right\|^{-1 /|\mathbf{m}|}\right\}} \leq \bar{\rho} .
$$

Since $\varepsilon>0$ is arbitrarily small, so finally we get

$$
\theta \leq \rho .
$$

Now, we will prove that $\theta \geq \rho$. If $\theta=\infty$, then there is nothing to prove. So let us assume that $0 \leq \theta<\infty$. Therefore, for a given $\varepsilon>0$ there exists $n_{0} \in \mathbb{N}$ such that, for all multi-indices $\mathbf{m}$ with $|\mathbf{m}|>n_{0}$, we have

$$
0 \leq \frac{\alpha(|\mathbf{m}|)}{\beta\left\{\log \left\|c_{\mathbf{m}}\right\|^{-1 /|\mathbf{m}|}\right\}}<\theta+\varepsilon=\bar{\theta}
$$

or

$$
\left\|c_{\mathbf{m}}\right\| \leq \exp \left[-|\mathbf{m}| \beta^{-1}\left\{\frac{\alpha(|\mathbf{m}|)}{\bar{\theta}}\right\}\right] .
$$

Now, from the property of maximum modulus (see [11], pp. 148), we have

$$
M(r, g) \leq \sum_{|\mathbf{m}|=0}^{\infty}\left\|c_{\mathbf{m}}\right\| k_{\mathbf{m}} r^{|\mathbf{m}|}
$$

or

$$
M(r, g) \leq \sum_{|\mathbf{m}|=0}^{\infty} k_{\mathbf{m}} r^{|\mathbf{m}|} \exp \left[-|\mathbf{m}| \beta^{-1}\left\{\frac{\alpha(|\mathbf{m}|)}{\bar{\theta}}\right\}\right] .
$$

On the lines of the proof of the theorem given by Srivastava and Kumar (see [7], Theorem 2.1, pp. 666), we get

$$
\rho \leq \theta \text {. }
$$

Combining this with inequality (28), we get (17). Hence, Theorem 6 is proved.

Next, we prove the following.

Theorem 7. Let $g: \mathbb{R}^{n+1} \rightarrow C l_{0 n}$ be a special monogenic function whose Taylor's series representation is given by (9). Also let $\alpha(x), \beta(x), \gamma(x) \in L^{0}$ and $0<\rho<\infty$; then the generalized type $\sigma$ of $g(z)$ is given as

$$
\sigma=\sigma(\alpha, \beta, \rho, g)=\lim _{|\mathbf{m}| \rightarrow \infty} \sup \frac{\alpha(|\mathbf{m}| / \rho)}{\beta\left[\left\{\gamma\left(e^{1 / \rho}\left\|c_{\mathbf{m}}\right\|^{-1 /|\mathbf{m}|}\right)\right\}^{\rho}\right]} .
$$


Proof. Write

$$
\eta=\lim _{|\mathbf{m}| \rightarrow \infty} \sup \frac{\alpha(|\mathbf{m}| / \rho)}{\beta\left[\left\{\gamma\left(e^{1 / \rho}\left\|c_{\mathbf{m}}\right\|^{-1 /|\mathbf{m}|}\right)\right\}^{\rho}\right]} .
$$

Now, first we prove that $\sigma \geq \eta$. From (16), for arbitrary $\varepsilon>0$ and all $r>r_{0}(\varepsilon)$, we have

$$
M(r, g) \leq \exp \left[\alpha^{-1}\left\{\bar{\sigma} \beta\left[\{\gamma(r)\}^{\rho}\right]\right\}\right],
$$

where $\bar{\sigma}=\sigma+\varepsilon$. Now, using (19), we get

$$
\left\|c_{\mathbf{m}}\right\| \leq \frac{1}{\sqrt{k_{\mathbf{m}}}} r^{-|\mathbf{m}|} \exp \left[\alpha^{-1}\left\{\bar{\sigma} \beta\left[\{\gamma(r)\}^{\rho}\right]\right\}\right]
$$

Now, as in the proof of Theorem 6, here this inequality reduces to

$$
\left\|c_{\mathbf{m}}\right\| \leq r^{-|\mathbf{m}|} \exp \left[\alpha^{-1}\left\{\bar{\sigma} \beta\left[\{\gamma(r)\}^{\rho}\right]\right\}\right] .
$$

Putting $r=\gamma^{-1}\left(\left[\beta^{-1}\{(1 / \bar{\sigma}) \alpha(|\mathbf{m}| / \rho)\}\right]^{1 / \rho}\right)$, we get

$$
\begin{aligned}
\left\|c_{\mathbf{m}}\right\| \leq & \left\{\gamma^{-1}\left(\left[\beta^{-1}\left\{\frac{1}{\bar{\sigma}} \alpha\left(\frac{|\mathbf{m}|}{\rho}\right)\right\}\right]^{1 / \rho}\right)\right\}^{-|\mathbf{m}|} \\
& \times \exp \left(\frac{|\mathbf{m}|}{\rho}\right)
\end{aligned}
$$

or

$$
\left\|c_{\mathbf{m}}\right\|^{-1 /|\mathbf{m}|} \geq\left\{\gamma^{-1}\left(\left[\beta^{-1}\left\{\frac{1}{\bar{\sigma}} \alpha\left(\frac{|\mathbf{m}|}{\rho}\right)\right\}\right]^{1 / \rho}\right)\right\} \exp \left(\frac{-1}{\rho}\right)
$$

or

$$
\frac{\alpha(|\mathbf{m}| / \rho)}{\beta\left[\left\{\gamma\left(e^{1 / \rho}\left\|c_{\mathbf{m}}\right\|^{-1 /|\mathbf{m}|}\right)\right\}^{\rho}\right]} \leq \bar{\sigma}
$$

Now, proceeding to limits as $|\mathbf{m}| \rightarrow \infty$, we get

$$
\eta \leq \bar{\sigma}
$$

Since $\varepsilon>0$ is arbitrarily small, so finally we get

$$
\eta \leq \sigma
$$

Now, we will prove that $\eta \geq \sigma$. If $\eta=\infty$, then there is nothing to prove. So let us assume that $0 \leq \eta<\infty$. Therefore, for a given $\varepsilon>0$ there exists $n_{0} \in \mathbb{N}$ such that, for all multi-indices m with $|\mathbf{m}|>n_{0}$, we have

$$
0 \leq \frac{\alpha(|\mathbf{m}| / \rho)}{\beta\left[\left\{\gamma\left(e^{1 / \rho}\left\|c_{\mathbf{m}}\right\|^{-1 /|\mathbf{m}|}\right)\right\}^{\rho}\right]} \leq \eta+\varepsilon=\bar{\eta}
$$

or

$$
\left\|c_{\mathbf{m}}\right\| \leq\left(\gamma^{-1}\left\{\left[\beta^{-1}\left\{\frac{1}{\bar{\eta}} \alpha\left(\frac{|\mathbf{m}|}{\rho}\right)\right\}\right]^{1 / \rho}\right\}\right)^{-|\mathbf{m}|} e^{|\mathbf{m}| / \rho} .
$$

Now, from the property of maximum modulus (see [11], pp. 148), we have

$$
M(r, g) \leq \sum_{|\mathbf{m}|=0}^{\infty}\left\|c_{\mathbf{m}}\right\| k_{\mathbf{m}} r^{|\mathbf{m}|}
$$

or

$$
\begin{aligned}
M(r, g) \leq \sum_{|\mathbf{m}|=0}^{\infty} & k_{\mathbf{m}} r^{|\mathbf{m}|} \\
& \times\left(\gamma^{-1}\left\{\left[\beta^{-1}\left\{\frac{1}{\bar{\eta}} \alpha\left(\frac{|\mathbf{m}|}{\rho}\right)\right\}\right]^{1 / \rho}\right\}\right)^{-|\mathbf{m}|} e^{|\mathbf{m}| / \rho} .
\end{aligned}
$$

On the lines of the proof of the theorem given by Srivastava and Kumar (see [7], Theorem 2.2, pp. 670), we get

$$
\sigma \leq \eta
$$

Combining this with (43), we get (34). Hence, Theorem 7 is proved.

Next, we have the following.

Theorem 8. Let $g: \mathbb{R}^{n+1} \rightarrow \mathrm{Cl}_{0 n}$ be a special monogenic function whose Taylor's series representation is given by (9). If $\alpha(x) \in \Lambda$ and $\beta(x) \in L^{0}$ then the generalized lower order $\lambda$ of $g(z)$ satisfies

$$
\lambda=\lambda(\alpha, \beta, g) \geq \lim _{|\mathbf{m}| \rightarrow \infty} \inf \frac{\alpha(|\mathbf{m}|)}{\beta\left\{\log \left\|c_{\mathbf{m}}\right\|^{-1 /|\mathbf{m}|}\right\}} .
$$

Further, if

$$
\psi(t)=\max _{|\mathbf{m}|=t}\left\{\frac{\left\|c_{\mathbf{m}}\right\| k_{\mathbf{m}}}{\left\|c_{\mathbf{m}^{\prime}}\right\| k_{\mathbf{m}^{\prime}}},\left|\mathbf{m}^{\prime}\right|=|\mathbf{m}|+1\right\}
$$

is a nondecreasing function of t, then equality holds in (49).

Proof. The proof of the above theorem follows on the lines of the proof of Theorem 6 and [7] Theorem 2.4 (pp. 674). Hence, we omit the proof.

Next, we have the following.

Theorem 9. Let $g: \mathbb{R}^{n+1} \rightarrow \mathrm{Cl}_{0 n}$ be a special monogenic function whose Taylor's series representation is given by (9). Also let $(x), \beta(x), \gamma(x) \in L^{0}$ and $0<\rho<\infty$; then the generalized lower type $\omega$ of $g(z)$ satisfies

$$
\omega=\omega(\alpha, \beta, \rho, g) \geq \lim _{|\mathbf{m}| \rightarrow \infty} \inf \frac{\alpha(|\mathbf{m}| / \rho)}{\beta\left[\left\{\gamma\left(e^{1 / \rho}\left\|c_{\mathbf{m}}\right\|^{-1 /|\mathbf{m}|}\right)\right\}^{\rho}\right]} .
$$

Further, if

$$
\psi(t)=\max _{|\mathbf{m}|=t}\left\{\frac{\left\|c_{\mathbf{m}}\right\| k_{\mathbf{m}}}{\left\|c_{\mathbf{m}^{\prime}}\right\| k_{\mathbf{m}^{\prime}}},\left|\mathbf{m}^{\prime}\right|=|\mathbf{m}|+1\right\}
$$

is a nondecreasing function of t, then equality holds in (51). 
Proof. The proof of the above theorem follows on the lines of the proof of Theorem 7 and [7] Theorem 2.4 (pp. 674). Hence, we omit the proof.

\section{Conflict of Interests}

The author declares that there is no conflict of interests regarding the publication of this paper.

\section{Acknowledgment}

The author is very thankful to the referee for the valuable comments and observations which helped in improving the paper.

\section{References}

[1] R. de Almeida and R. S. Kraußhar, "On the asymptotic growth of entire monogenic functions," Zeitschrift für Analysis und ihre Anwendungen, vol. 24, no. 4, pp. 791-813, 2005.

[2] D. Constales, R. de Almeida, and R. S. Krausshar, "On the growth type of entire monogenic functions," Archiv der Mathematik, vol. 88, no. 2, pp. 153-163, 2007.

[3] D. Constales, R. de Almeida, and R. S. Krausshar, "On the relation between the growth and the Taylor coefficients of entire solutions to the higher-dimensional Cauchy-Riemann system in $\mathbb{R}^{n+1}$," Journal of Mathematical Analysis and Applications, vol. 327, no. 2, pp. 763-775, 2007.

[4] M. A. Abul-Ez and D. Constales, "Basic sets of polynomials in Clifford analysis," Complex Variables: Theory and Application, vol. 14, no. 1-4, pp. 177-185, 1990.

[5] M. A. Abul-Ez and R. De Almeida, "On the lower order and type of entire axially monogenic functions," Results in Mathematics, vol. 63, no. 3-4, pp. 1257-1275, 2013.

[6] M. N. Seremeta, "On the connection between the growth of the maximum modulus of an entire function and the moduli of the coefficients of its power series expansion," The American Mathematical Society Translations, vol. 88, no. 2, pp. 291-301, 1970.

[7] G. S. Srivastava and S. Kumar, "On the generalized order and generalized type of entire monogenic functions," Demon Math, vol. 46, no. 4, pp. 663-677, 2013.

[8] S. Kumar and K. Bala, "Generalized type of entire monogenic functions of slow growth," Transylvanian Journal of Mathematics and Mechanics, vol. 3, no. 2, pp. 95-102, 2011.

[9] S. Kumar and K. Bala, "Generalized order of entire monogenic functions of slow growth," Journal of Nonlinear Science and its Applications, vol. 5, no. 6, pp. 418-425, 2012.

[10] S. Kumar and K. Bala, "Generalized growth of monogenic Taylor series of finite convergence radius," Annali dell'Universitá di Ferrara VII: Scienze Matematiche, vol. 59, no. 1, pp. 127-140, 2013.

[11] M. A. Abul-Ez and D. Constales, "Linear substitution for basic sets of polynomials in Clifford analysis," Portugaliae Mathematica, vol. 48, no. 2, pp. 143-154, 1991. 


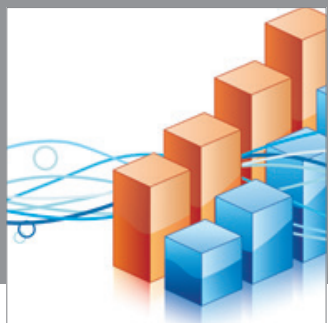

Advances in

Operations Research

mansans

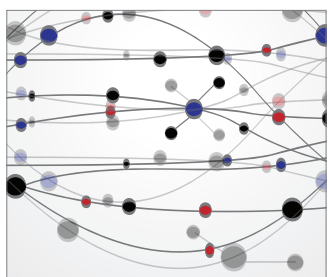

The Scientific World Journal
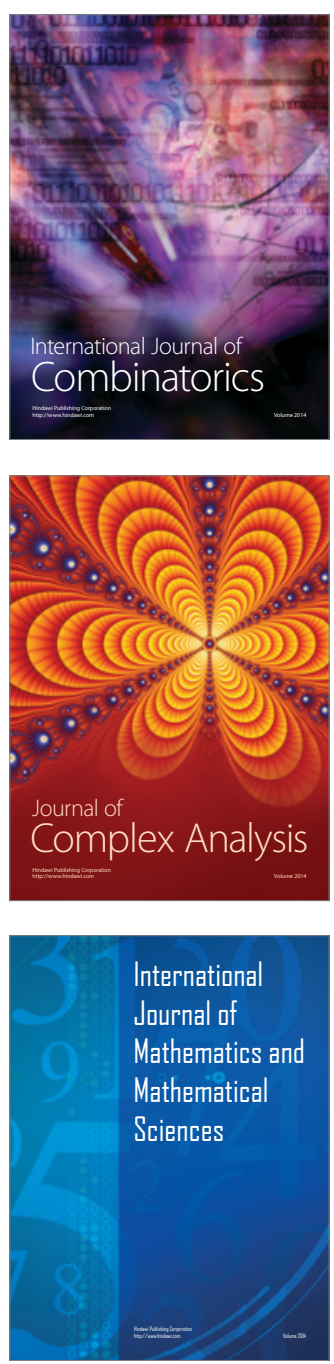
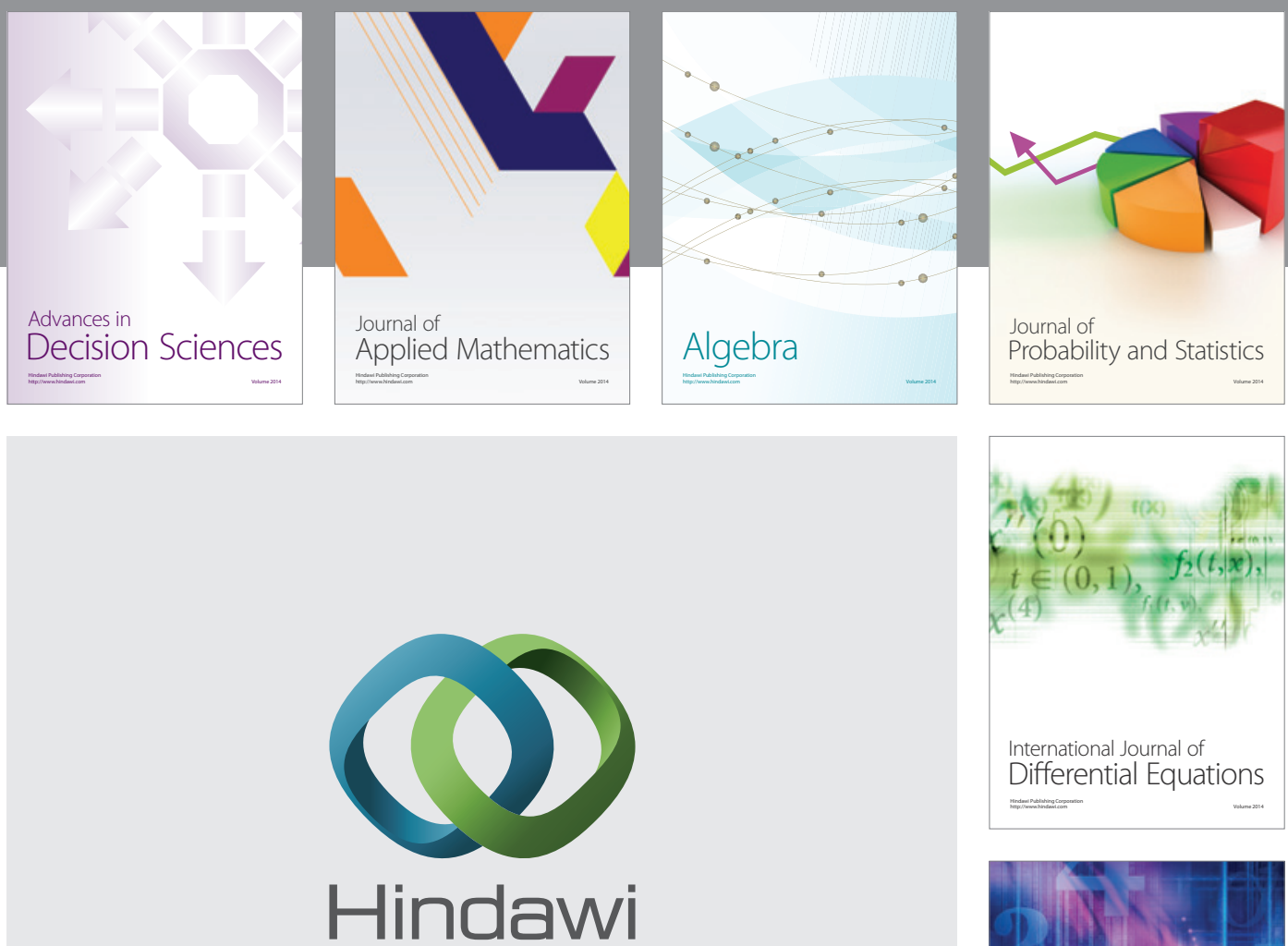

Submit your manuscripts at http://www.hindawi.com
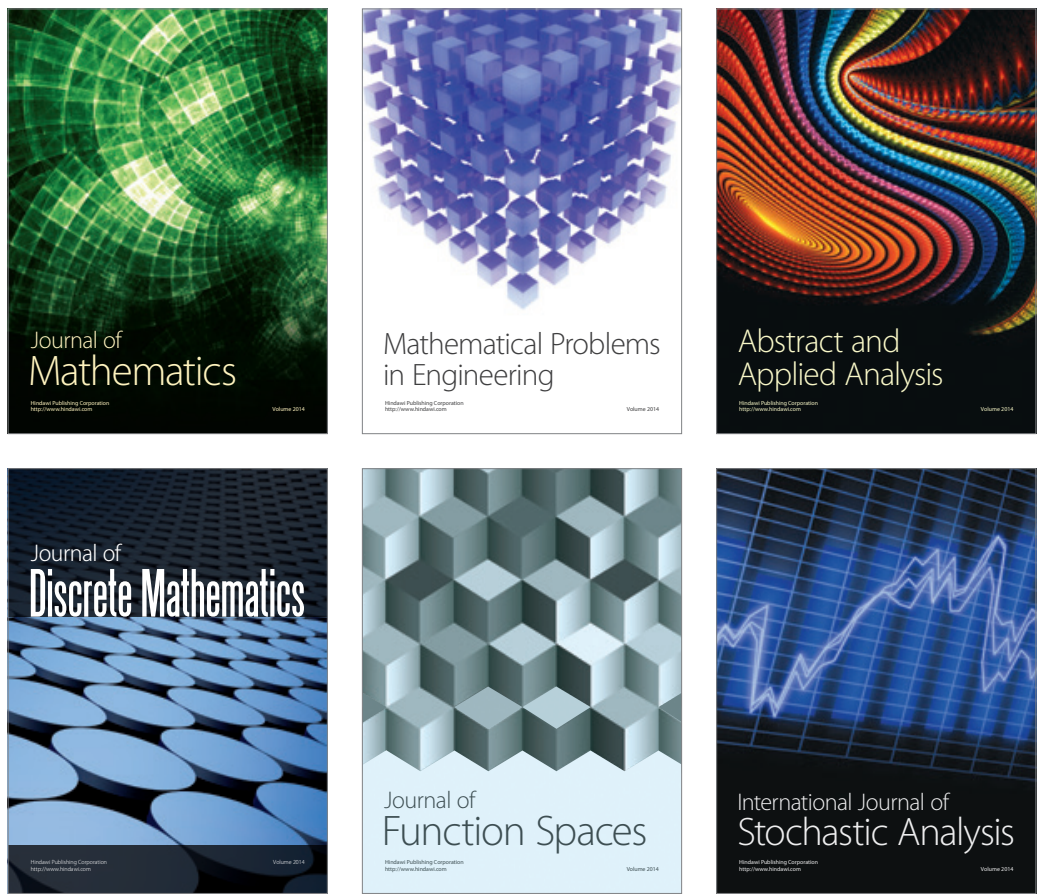

Journal of

Function Spaces

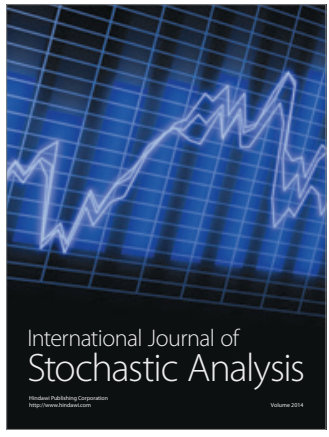

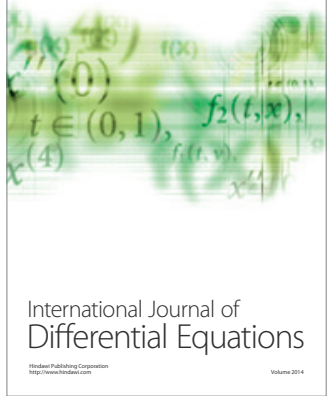
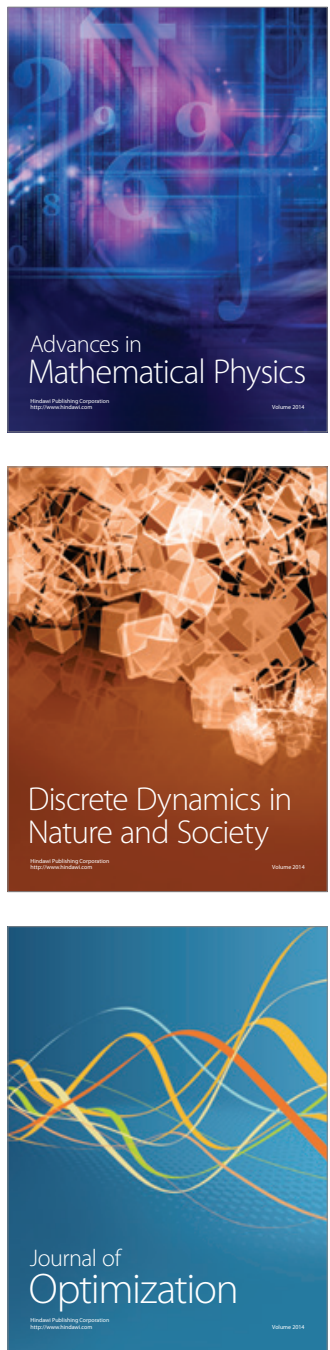